\title{
Non-Invasive Characterization of Atrial Arrhythmic Driving Mechanisms in Computer Models
}

\author{
Victor G Marques $^{1}$, Miguel Rodrigo ${ }^{2}$, María S Guillem ${ }^{2}$, João Salinet ${ }^{1}$ \\ ${ }^{1}$ Federal University of ABC, São Bernardo do Campo, Brazil \\ 2 ITACA Institute, Universitat Politecnica de Valencia, Spain
}

\begin{abstract}
Atrial tachycardia (AT), atrial flutter (AFL) and atrial fibrillation (AF) are among the most common cardiac arrhythmias and are driven by localized sources (ectopic focus in AT, macro-reentrant circuit in AFL and rotors in AF) that can be targeted for ablation. We aimed to characterize such mechanisms from the non-invasive perspective of body surface potential mapping (BSPM), using realistic computer models. Dominant frequency (DF) maps were studied to estimate the frequency of the driving mechanism and to analyze its spatio-temporal distribution of this frequency. Singularity points (SPs) were detected in phase maps and their distribution and rotational patterns were compared between arrhythmias. The driver frequencies were more expressed on the anterior portion of the torso for the right atrium and on the posterior portion for the left atrium. Rotational activity was detected in all arrhythmias, with increasing levels of spatial-temporal stability (AF, AT and AFL, respectively). These results can be used to identify the driving mechanisms and, in the future, to locate them in the atria.
\end{abstract}

\section{Introduction}

Supraventricular tachyarrhythmias, including focal atrial tachychardia (AT), atrial flutter (AFL) and atrial fibrillation (AF), are among the most common cardiac arrhythmias in the world and can pose serious risks, such as an increased chance of thromboembolic events [1].

These arrhythmias are driven by localized sources, which can be targeted in radio-frequency ablation therapy in order to restore sinus rhythm [1]: AT is maintained by ectopic foci. AFL is driven by a macro-reentrant circuit, localized typically, but not obligatorily, around the cavotricuspid isthmus. AF has been shown to be maintained by ectopic, re-entrant circuits and functional rotors, which appear preferably when structural and/or functional atrial remodeling occurs [2].

The detection of these driving mechanism and their location is crucial to the success of ablation therapies.
This is usually done via an electrophysiological study, which relies on catheter mapping and several signal processing techniques in order to find the driving source(s) [3]. Non-invasive identification and localization of the driving sources using body surface potential mapping (BSPM) might be an important auxiliary tool to be used in clinical practice prior catheter ablation, reducing procedural time and their associated risks [3].

This study aimed to characterize non-invasively three atrial arrhythmia mechanisms - ectopic activity (AT), macro-reentrant circuit (AFL) and functional reentry (AF) - generated by realistic mathematical models in order to differentiate them from the perspective of BSPM.

\section{Methods}

The methodology is summarized in the block diagram in Fig. 1. Computer generated BSPMs representing the different arrhythmic mechanisms were processed in time, frequency and phase domains, obtaining maps from which specific characteristics were extracted. All analyses were made in Python 3.6.

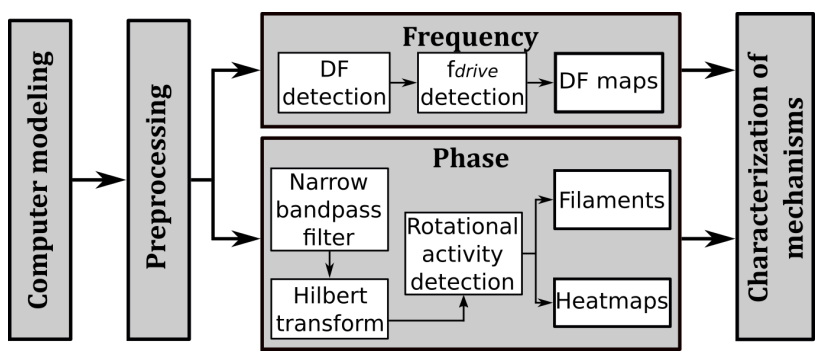

Figure 1. General block diagram

\subsection{Computer modeling}

A realistic three-dimensional model of the atrial anatomy composed by 284578 nodes and 1353783 tetrahedrons ( $673.4 \pm 130.3 \mu \mathrm{m}$ between nodes) was used to simulate the electrical behavior of the atria in arrhythmic conditions. 19 simulations represented arrhythmias 
originated by three distinct mechanisms: AT (4 simulations) driven by an ectopic focus; AFL (4 simulations) driven by a macro-reentrant circuit; AF (11 simulations) driven by functional rotors [4]. BSPMs were obtained by solving the forward problem with the boundary element method (sampling frequency $=500 \mathrm{~Hz}$ ), resulting in 771 data points, of which 567 points were selected to represent a realistic measurement (excluding points inside the waist, neck or arms).

\subsection{Preprocessing}

Data points were reshaped into a two-dimensional configuration by projecting their positions on a cylinder. The cylinder was unwrapped and the points were interpolated into a $30 \times 65$ grid using cubic splines. A 4 th order band-pass Butterworth filter was applied between 2 and $20 \mathrm{~Hz}$, covering the spectral components related to the atrial arrhythmic mechanisms [5].

\subsection{Frequency analysis}

The frequencies of the driver mechanisms ( $\left.f_{\text {drive }}\right)$ were estimated using a combination of analyses in time and frequency with spatial information from the BSPM.

Activation times were detected using a wavelet transform modulus maxima approach [6]. Average activation frequency was estimated as the inverse of the average interval between activation times. The spectral content for each data point was estimated via Welch periodograms (segments of $2.7 \mathrm{~s}$, with Hanning window, zero-padded to 2048 points and with $90 \%$ overlap; frequency resolution of $0.37 \mathrm{~Hz}$ ). The dominant frequency (DF) of the spectrum was defined as the peak closest to the average activation frequency, in order to reduce the detection harmonics of the driver frequency.

The $f_{\text {drive }}$ was defined as the highest dominant frequency (HDF) along the torso after applying a spatial mask disconsidering $2 \%$ of the highest DF values to determine the HDF, thus avoiding harmonics. Estimated $f_{\text {drive }}$ were compared with the original modeled frequency of the driver mechanism by calculating the absolute error.

The distributions of the DFs on the torso maps were analyzed by calculating the BSPM DF range (HDF-lowest $D F$ ) and identifying regions on the BSPM where the $f_{\text {drive }}$ is expressed, defined as connected portions of the torso with DF values in the range $f_{\text {drive }} \pm 0.2 \mathrm{~Hz}$. Binary maps ( $t r u e ~=f_{\text {drive }}$ regions) were used to create percentage maps indicating how many models (divided by atrium) had $f_{\text {drive }}$ expressed in each torso location. Average number and sizes of the regions were used to compare the arrhythmias and identify patterns in spectral organization.

\subsection{Phase analysis}

A 4th order Butterworth narrowband-pass filter $( \pm 1 \mathrm{~Hz})$ was applied on the BSPM signals around $f_{\text {drive }}$ with the objective of stabilizing the atrial activity seen on the surface, especially for AF [7]. The signals were then downsampled to $128 \mathrm{~Hz}$ to decrease computation time. Phase was determined via Hilbert transform, defined as the ratio between imaginary and real portions of the results [4].

Phase maps were generated in order to detect singularity points (SPs), defined as the points around which all the phases converge [6], using a method based on edge detection: endpoints of edges detected by Canny's method were defined as SP candidates [8].

The detection of the SPs is based on the phase progression along 5 rings with varying radii $(\approx 2.2$ to 13.2 $\mathrm{cm}$ ) around the SP candidates. Three criteria must be attended in at least two rings for a SP to be detected: the phase should progress in a range of at least $\pi$, the progression should be at least $60 \%$ ordered and there should be no phase leaps larger than $\pi$ [4].

The spatio-temporal distribution of the SPs was analyzed based on filament maps and heatmaps. A filament is defined as the connection of the SPs in phase maps along subsequent time instants, around which at least one full cycle of rotation was sustained. Threedimensional connected component analysis was applied using a kernel of shape 3x3x64 $(6.36 \mathrm{~cm}$ x $6.63 \mathrm{~cm}$ $\mathrm{x} 0.5 \mathrm{~s}$ ) to connect and differentiate the individual filaments, with any two SPs belonging to the same region being assigned to the same filament. Heatmaps are the histogram of the SPs belonging to filaments along time.

The number of cycles and the frequency of filaments were estimated from the edge maps. Regions in heatmaps concentrating SPs were segmented with connected component analysis; the number, size and density (i.e. \% of SPs inside region / Number of points inside region) of each region were calculated, in addition to the number of SPs per second.

\subsection{Statistical analysis}

Anderson-Darling normality test was applied to determine the normality of the data. Homogeneity of variances was evaluated using Bartlett's test. If both the normality and homoscedasticity criteria were followed, analysis of variances followed by Tukey's range test was used. Otherwise, Kruskal-Wallis H-test, followed by Dunn's multiple comparison test, were used. Significance was set at $p<0.05$ and significant differences are marked with a ${ }^{*}$; values are given in mean \pm se or mean \pm std where indicated with a $†$. 


\section{Results}

\subsection{Frequency characterization}

$f_{\text {drive }}$ was estimated with an absolute error of $0.06 \pm$ $0.06 \mathrm{~Hz}, 0.18 \pm 0.14 \mathrm{~Hz}$ and $0.84 \pm 0.99 \mathrm{~Hz}$ for AT, AFL and AF respectively. The characteristics of the regions expressing $f_{\text {drive }}$ are given in Table 1 . AF presents a significantly higher number of regions expressing $f_{\text {drive }}$, but the portion of the torso occupied by these regions was significantly smaller than in the other two arrhythmias $(p<0.05)$, where this frequency is expressed in almost the totality of the torso. DF range is significantly larger in AF than in AFL or AT $(p<0.05)$. The distribution of the regions expressing $f_{\text {drive }}$ is displayed in Fig. 2 for drivers in the left and right atria (LA and RA, respectively).

Table 1. Characteristics of DF expression on torso

\begin{tabular}{cccc}
\hline & $\begin{array}{c}\text { \# of } f_{\text {drive }} \\
\text { regions } \dagger\end{array}$ & $\begin{array}{c}f_{\text {drive }} \text { region } \\
\text { size (\% of torso) }\end{array}$ & $\begin{array}{c}\text { DF range } \\
\text { (Hz) } \dagger\end{array}$ \\
\hline AT & $1.50 \pm 0.50$ & $77.96 \pm 20.96$ & $0.76 \pm 0.70$ \\
AFL & $1.00 \pm 0.00$ & $97.62 \pm 2.10$ & $0.24 \pm 0.49$ \\
AF & $2.64 \pm 0.43 *$ & $22.27 \pm 10.63 *$ & $2.43 \pm 0.78 *$ \\
\hline
\end{tabular}

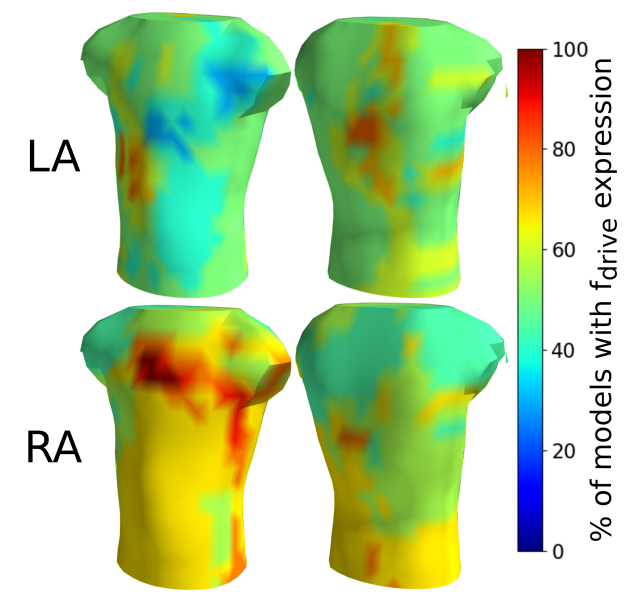

Figure 2. Distribution of estimated $f_{\text {drive }}$ regions for the models in the LA (top) and RA (bottom)

\subsection{Phase characterization}

The number of filaments per second, their duration and frequency of rotation are given in Table 2. No significant differences were encountered when comparing the number of filaments per second; filament duration was significantly shorter in AF than in the other two arrhythmias $(p<0.05)$. The most temporally stable filaments appeared in AFL, followed by AT. Average frequency of rotation around filaments is within $0.5 \mathrm{~Hz}$ of $f_{\text {drive }}$ in
$73.68 \%$ and within $1.0 \mathrm{~Hz}$ in $89.47 \%$ of the models. Fig. 3 shows examples of filaments for all the arrhythmias.

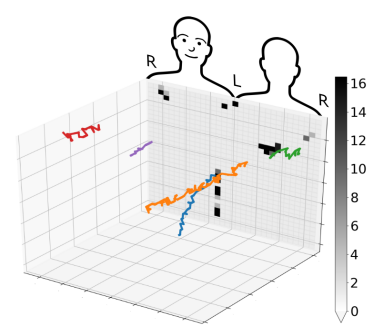

a) AT (ectopic focus in IVC)

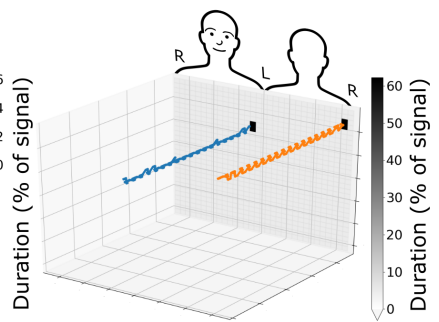

b) Typical CW AFL

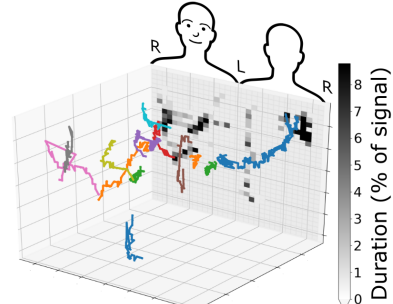

c) AF (rotor in RIPV)

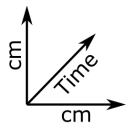

Figure 3. Representative examples of filaments and heatmaps in AT (a), AFL (b) and AF(c). Each color represents a different filament. IVC: inferior vena cava; CW: clockwise; RIPV: right inferior pulmonary vein

Table 2. Characteristics of the filaments for each model and arrhythmia group

\begin{tabular}{cccc}
\hline & $\begin{array}{c}\text { Filaments } \\
\text { per second } \dagger\end{array}$ & $\begin{array}{c}\text { Duration } \\
\text { (\% of signal) }\end{array}$ & $\begin{array}{c}\text { Frequency } \\
\text { (Hz) }\end{array}$ \\
\hline AT & $1.25 \pm 0.57$ & $78.42 \pm 13.80$ & $3.96 \pm 0.02$ \\
AFL & $0.96 \pm 0.62$ & $90.98 \pm 9.02$ & $4.18 \pm 0.11$ \\
AF & $1.16 \pm 0.65$ & $38.40 \pm 10.42 *$ & $5.59 \pm 0.19 *$ \\
\hline
\end{tabular}

The characteristics of SP regions in heatmaps are given in Table 3. While no differences in the number of regions was found ( $p=0.11$ ), their sizes are significantly smaller in AF than AFL $(p<0.01)$. There was no significant difference in the number of SPs per second; differences appear between AF and AFL in the density of SPs $(p<0.01)$, with AFL presenting higher values, indicative of higher spatio-temporal stability. AT presents intermediate values of region size and SP density between the other two arrhythmias. The distribution of SPs in heatmaps is displayed in Fig. 4 for the models in the LA and RA.

Table 3. Characteristics of the SP regions in heatmaps

\begin{tabular}{ccccc}
\hline & $\begin{array}{c}\text { \# of SP } \\
\text { clusters } \dagger\end{array}$ & $\begin{array}{c}\text { Cluster size } \\
\text { (\% of torso) }\end{array}$ & $\begin{array}{c}\text { SP } \\
\text { density }\end{array}$ & SPs/s $\dagger$ \\
\hline AT & $4.25 \pm 2.06$ & $0.64 \pm 0.14 *$ & $2.25 \pm 0.48$ & $2.13 \pm 1.25$ \\
AFL & $2.00 \pm 0.00 *$ & $0.44 \pm 0.23 *$ & $10.79 \pm 2.71 *$ & $1.88 \pm 0.18$ \\
AF & $10.64 \pm 7.00 *$ & $0.85 \pm 0.14 *$ & $0.62 \pm 0.11 *$ & $1.36 \pm 0.56$ \\
\hline
\end{tabular}




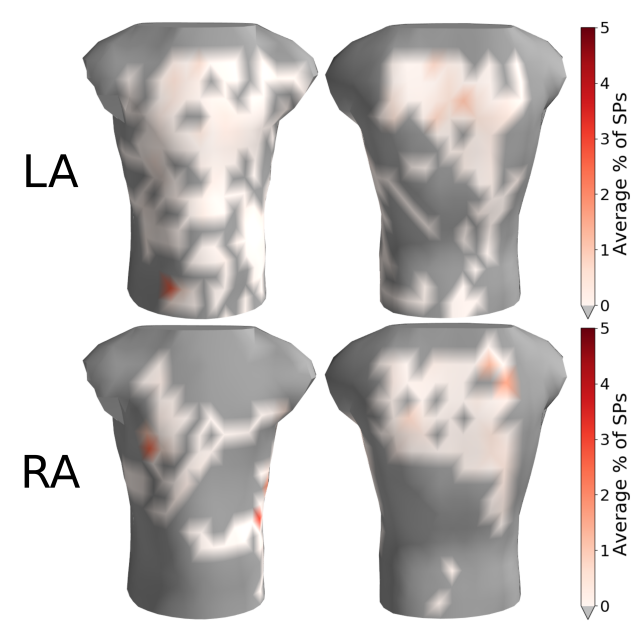

Figure 4. Distribution of SPs on the torso, for the models in the LA (top) and RA (bottom)

\section{Discussion and Conclusions}

In this work, analyses performed in the time, frequency and phase domains were used to characterize the BSPM patterns from three different atrial arrhythmia mechanisms. We developed an unified methodology to estimate the driver mechanism's frequency and extended the phase analysis to encompass all three arrhythmias, thus allowing the characterization and direct comparison of the mechanisms non-invasively.

The distribution of the DFs on the BSPM was almost uniform for TA and AFL, while AF had more variant patterns with larger DF ranges, highlighting its complexity. The distribution of $f_{\text {drive }}$ on the torso followed similar patterns as in other studies [3,9], with most RA models presenting $f_{\text {drive }}$ regions on the anterior portion of the torso and most LA models, on the posterior portion.

It this study, stable rotors on BSPM were detected not only in AF and AFL, as reported before [7, 10], but also in AT. Filaments were the most stable in AFL, where they were concentrated in few regions with high number of SPs, which can be explained by the anchoring of macroreentrant circuits in anatomical structures. AT presented filaments with intermediate duration and stability when compared to the other arrhythmias, even though the driving mechanism is an ectopic focus, generating centrifugal wavefronts. While other similar works did not detect this behavior [4], our configuration to analyze the phase progression around SPs revealed relevant patterns along circles with radii larger than $2 \mathrm{~cm}$.

Average filament duration in AF was much shorter than in the other two arrhythmias. This is related to the limited field of view of the BSPM, which can hide stable rotors, to the spatial resolution of the BSPM and to the large number of shorter filaments, unique in AF models.
The current results allow the identification of specific patterns for each driving mechanisms, which could be further explored before patient treatment.

\section{Acknowledgments}

This work was financed by the São Paulo Research Foundation (FAPESP), grant number 2017/19775-3.

\section{References}

[1] Issa ZF, Miller JM, Zipes DP. Clinical arrhythmology and electrophysiology: a companion to Braunwaldś heart disease. Elsevier Health Sciences, 2009.

[2] Narayan SM, et al. Treatment of atrial fibrillation by the ablation of localized sources: CONFIRM (conventional ablation for atrial fibrillation with or without focal impulse and rotor modulation) trial. Journal of the American College of Cardiology 2012;60(7):628-636.

[3] Bojarnejad M, et al. Non-invasive estimation of left atrial dominant frequency in atrial fibrillation from different electrode sites: insight from body surface potential mapping. Journal of atrial fibrillation 2014;7(3).

[4] Rodrigo M, et al. Technical considerations on phase mapping for identification of atrial reentrant activity in direct-and inverse-computed electrograms. Circulation Arrhythmia and Electrophysiology 2017;10(9):e005008.

[5] Guillem MS, et al. Noninvasive mapping of human atrial fibrillation. Journal of cardiovascular electrophysiology 2009;20(5):507-513.

[6] Vijayakumar R, et al. Methodology considerations in phase mapping of human cardiac arrhythmias. Circulation Arrhythmia and Electrophysiology 2016; 9(11):e004409.

[7] Rodrigo M, et al. Body surface localization of left and right atrial high-frequency rotors in atrial fibrillation patients: a clinical-computational study. Heart Rhythm 2014;11(9):1584-1591.

[8] Salinet J, et al. Propagation of meandering rotors surrounded by areas of high dominant frequency in persistent atrial fibrillation. Heart rhythm 2017;14(9):1269 1278.

[9] Guillem MS, et al. Noninvasive localization of maximal frequency sites of atrial fibrillation by body surface potential mapping. Circulation Arrhythmia and Electrophysiology 2013;6(2):294-301.

[10] Liberos A, et al. Phase singularity point tracking for the identification of typical and atypical flutter patients: A clinical-computational study. Computers in biology and medicine 2019;104:319-328.

Address for correspondence:

Victor Gonçalves Marques

Biomedical Engineering - CECS

Federal University of ABC

E-mail: vgmarques@ufabc.edu.br 